\title{
Effects of the Lysine Methyltransferase Inhibitor AZ505 on Bone Metabolism
}

\author{
Min-Kyoung Song 1,2, Suhan Jung', Seojin Hong', Jun-Oh Kwon', Min Kyung Kim', Hong-Hee Kim ${ }^{1}$ \\ 'Department of Cell and Developmental Biology, Dental Research Institute, School of Dentistry, Seoul National University, Seoul; \\ ${ }^{2}$ Department of Internal Medicine, Seoul National University Hospital, Seoul, Korea
}

Corresponding author

Hong-Hee Kim

Department of Cell and Developmental

Biology, School of Dentistry, Seoul National

University, 101 Daehak-ro, Jongno-gu,

Seoul 03080, Korea

Tel: +82-2-740-8686

Fax: +82-2-763-3613

E-mail: hhbkim@snu.ac.kr

Received: June 18, 2021

Revised: August 20, 2021

Accepted: September 13, 2021
Background: Protein methylation has important role in regulating diverse cellular responses, including differentiation, by affecting protein activity, stability, and interactions. AZ505 is an inhibitor of the SET and MYND domain-containing protein 2 lysine methylase. In this study, we investigated the effect of AZ505 on osteoblast and osteoclast differentiation in vitro and evaluated the effect of AZ505 in vivo on the long bones in mice. Methods: Osteoblast differentiation was assessed by alkaline phosphatase (ALP) and Alizarin red staining after culturing calvarial preosteoblasts in an osteogenic medium. Osteoclast differentiation was analyzed by tartrate-resistant acid phosphatase (TRAP) staining in bone marrow-derived macrophages cultured with macrophage-colony stimulating factor and receptor activator of nuclear factor-KB ligand (RANKL). For in vivo experiments, mice were intraperitoneally injected with AZ505 and femurs were examined by micro-computed tomography. Results: AZ505 increased ALP and Alizarin red staining in cultured osteoblasts and the expression of osteoblast marker genes, including RunX2 and osteocalcin. AZ505 resulted in decreased TRAP-staining of osteoclasts and expression of c-Fos and nuclear factor of activated T cells transcription factors and osteoclast marker genes, including cathepsin $\mathrm{K}$ and dendritic cell-specific transmembrane protein. Unexpectedly, in vivo administration of AZ505 markedly decreased the trabecular bone mass of femurs. In support of this catabolic result, AZ505 strongly upregulated RANKL expression in osteoblasts. Conclusions: The results indicate that AZ505 has a catabolic effect on bone metabolism in vivo despite its anabolic effect in bone cell cultures. The findings indicate that cell culture data should be extrapolated cautiously to in vivo outcomes for studying bone metabolism.

Key Words: Bone density · Methyltransferases · Osteoblast · Osteoclast

\section{INTRODUCTION}

Epigenetic regulation of gene expression has been recognized as a crucial event

Copyright (C) 2021 The Korean Society for Bone and Mineral Research

This is an Open Access article distributed under the terms of the Creative Commons Attribution Non-Commercial License (https://creativecommons.org/licenses/by-nc/4.0/) which permits unrestricted non-commercial use, distribution, and reproduction in any medium, provided the original work is properly cited.

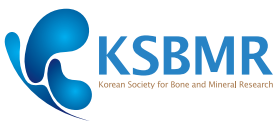

in physiological and pathological development, regeneration, and repair that involves cell differentiation.[1,2] Protein methylation/demethylation is an important division in the epigenetic control of gene expression. Histone lysine methyltransferases and demethylases modulate methylation dynamics of specific lysine residues of histones at promoters of target genes. SET and MYND domain-containing protein 2 (SMYD2) is a methyltransferase that methylates histone $\mathrm{H} 3$ at lysine 4 (H3K4) and lysine 36 (H3K36).[3] Dimethylation of H3K36 by SMYD2 at tumor ne- 
crosis factor-a (TNF-a) promoter suppresses TNF-a expression in macrophages.[4] Besides histones, other proteins are also substrates of methyltransferases and demethylases. Lysine methylation of non-histone proteins has been reported to play critical roles in tumorigenesis by affecting modification of other proteins, protein-protein interaction, protein stability, and subcellular localization.[5] SMYD2 methylates p53 and Rb tumor suppressors resulting in the repression of their activities and consequent tumor promotion.[6-8] In addition, estrogen receptor-a (ER-a) was shown to be a target of SMYD2.[9,10] ER-a methylation at lysine 266 by SMYD2 leads to repression of ER-a-dependent transactivation. Despite the crucial involvements of epigenetics and ER signaling in the control of bone metabolism, the role of SMYD2 in the regulation of bone cells or bone mass has not been explored to date.

AZ505 has been developed as an inhibitor of protein methyltransferases with a great selectivity toward SMYD2. $[11,12]$ This chemical was shown to suppress the proliferation of breast cancer cells in vitro and tumor growth in vivo. [13] AZ505 also decreased cyst growth in mice model of autosomal dominant polycystic kidney disease.[14] These effects were attributed to the inhibition of SMYD2-mediated methylation of STAT3 and p65 transcription factors. In immune responses to Leishmania donovani, AZ505 increased pro-inflammatory response in infected J774 macrophages and multiplication of the pathogen in $B A L B / C$ mice by inhibiting epigenetic suppression of TNF-a expression SMYD2.[4]

In this study, we sought to evaluate the potential effect of AZ505 on the differentiation of 2 major types of bone cells; osteoblasts and osteoclasts. In addition, the in vivo effect of AZ505 on bone metabolism was assessed in mice.

\section{METHODS}

\section{Animals}

Eight-week-old female mice (C57BL/6) were used for intraperitoneal injection of AZ505. Five-week-old ICR male mice and neonatal ICR mice were used to prepare bone marrow-derived macrophages (BMMs) and calvarial preosteoblasts, respectively. All mice were purchased from OrientBio (Gapyeong, Korea). All animal experimental procedures were approved by the Institutional Animal Care and Use Committee of Seoul National University.

\section{Reagents}

AZ505 was purchased from MedChemExpress (Monmouth Junction, NJ, USA). Recombinant human macrophage colony-stimulating factor (M-CSF) and human soluble receptor activator of nuclear factor-KB ligand (RANKL) were purchased from PeproTech (Rocky Hill, NJ, USA). Antinuclear factor of activated T cells (NFATc1; 7A6) and anti-cFos (H125) antibodies were from Santa Cruz Biotechnology (Santa Cruz, CA, USA), and monoclonal antibodies against $\beta$-actin (AC-74) and secondary antibodies were purchased from Sigma-Aldrich (St. Louis, MO, USA). All other reagents were obtained from Sigma-Aldrich.

\section{Osteoclast differentiation}

Osteoclast differentiation culture was performed with mouse BMMs as previously described. $[15,16]$ In brief, bone marrow cells were flushed from the tibiae and femurs of 5-week-old female ICR mice and cultured in a-minimal essential medium (a-MEM) containing 10\% fetal bovine serum (FBS) overnight on culture dishes. Adherent cells were discarded, and nonadherent cells were further incubated with $30 \mathrm{ng} / \mathrm{mL}$ M-CSF in Petri dishes for 3 days. Adherent cells were harvested and used as BMMs. BMMs were seeded at $3 \times 10^{4}$ per well in 48 -well plates or $3 \times 10^{5}$ per well in 6-well plates and cultured with $30 \mathrm{ng} / \mathrm{mL}$ M-CSF. From the following day, cells were cultured with $30 \mathrm{ng} / \mathrm{mL}$ M-CSF and $100 \mathrm{ng} / \mathrm{mL}$ RANKL to induce osteoclast differentiation. To evaluate the differentiation extent, cells were fixed with $3.7 \%$ formaldehyde and permeabilized in $0.1 \%$ Triton $\mathrm{X}$-100 for $1 \mathrm{~min}$. Tartrate-resistant acid phosphatase (TRAP) staining was performed for 5 to $15 \mathrm{~min}$.

\section{Osteoblast differentiation}

Primary calvarial preosteoblasts were prepared from calvarae of neonatal mice, as previously described.[17] For the induction of osteogenesis, calvarial preosteoblasts were seeded at $3 \times 10^{4}$ per well in 48 -well plates or $3 \times 10^{5}$ per well in 6-well plates in a-MEM containing $10 \%$ FBS. On the next day, the medium was changed to an osteogenic medium containing $10 \mathrm{mM}$ of $\beta$-glycerophosphate and $100 \mu \mathrm{g} / \mathrm{mL}$ of ascorbic acid. To evaluate the differentiation extent, cells were stained for alkaline phosphatase (ALP) activity or the cell lysates were subjected to quantitative ALP activity assay. Matrix mineralization was assessed by Alizarin red staining. For quantitation, stained cells were 
incubated with $100 \mathrm{mM}$ of cetylpyridinium chloride for 2 $\mathrm{hr}$ at $37^{\circ} \mathrm{C}$ and the optical density of eluates was measured at $415 \mathrm{~nm}$.

\section{Co-culture}

Calvarial preosteoblasts $\left(2 \times 10^{4}\right)$ and bone marrow cells $\left(2 \times 10^{5}\right)$ were seeded on a collagen-coated 48-well plates and cultured with a-MEM containing $10 \%$ FBS in the presence of $10 \mathrm{nM}$ 1,25-dihydroxyvitamin D3 and $1 \mu \mathrm{M}$ prostaglandin $E_{2}$. The medium was changed every 3 days. To evaluate osteoclast formation, cells were fixed with 3.7\% formaldehyde and permeabilized in $0.1 \%$ Triton X-100 for $5 \mathrm{~min}$. TRAP staining was performed for 5 to $15 \mathrm{~min}$.

\section{Reverse transcription-quantitative polymerase chain reaction ( $\mathrm{RT}-\mathrm{qPCR}$ )}

Quantification of mRNA expression by real-time PCR analysis was carried out as previously described.[18] The primer sequences used were as follows: cbfa1, forward 5'CGC ACG ACA ACC GCA CCA-3' and reverse 5'-CAG CAC GGA GCA CAG GAA GTT-3'; sp7, forward 5'-ATG CGA AGC CTT GCC GTA CA-3' and reverse 5'-TCT ACC TGC GAC TGC CCC AA-3'; bglap, forward 5'-CCG GGA GCA GTG TGA GCT TA-3' and reverse 5'-TAG ATG CGT TTG TAG GCG GTC-3'; alpl, forward 5'-TCC GTG GGC ATT GTG ACT AC-3' and reverse 5'-TGG TGG CAT CTC GTT ATC CG-3'; col1a1, forward 5'-GCA TGG CCA AGA AGA CAT CC-3' and reverse $5^{\prime}$-CCT CGG GTT TCC ACG TCT-3'; ibsp, forward 5'- CAG AGG AGG CAA GCG TCA CT-3' and reverse 5'-CTG TCT GGG TGC CAA CAC TG-3'; nfatc1, forward 5'-CCAGTATACCAGCTCTGCCA-3' and reverse $5^{\prime}$-GTG GGA AGT CAG AAG TGG GT-3'; fos, forward 5'-ACT TCT TGT TTC CGG C-3', reverse 5'-AGC TTC AGG GTA GGT G-3'; acp5, forward 5'-CGA CCA TTG TTA GCC ACA TAC G-3' and reverse 5'-TCG TCC TGA AGA TAC TGC AGG TT3'; ctsk, forward 5'-ATA TGT GGG CCA CCA TGA AAG TT-3' and reverse 5'-TCG TTC CCC ACA GGA ATC TCT-3'; dcstamp, forward 5'-GGG TGC TGT TTG CCG CTG-3' and reverse 5'CGA CTC CTT GGG TTC CTT GCT-3'; tnfsf11, forward 5'-TGG AAG GCT CAT GGT TGG AT-3' and reverse 5'-CAT TGA TGG TGA GGT GTG CAA-3'; tnfsf1 1 $b$, forward 5'-GCA GCC CAG TGT GCA AGG AA-3' and reverse 5'-TTC GAT CTC CAG GTA ACG CCC-3'; hprt, forward 5'-CCT AAG ATG AGC GCA AGT TGA A-3' and reverse 5'-CCA CAG GGA CTA GAA CAC CTG CTA A-3'.

\section{Cell viability assay}

Cells were seeded in 48-well plates at the density of $3 \times 10^{4}$ cells per well and cultured in a differentiation medium together with vehicle or AZ505. On day 3, cells were subjected to cytotoxicity assay using the QuantiMax ${ }^{\mathrm{TM}}$ WST-8 assay kit (Biomax, Seoul, Korea). After incubation with the reagents for $1 \mathrm{hr}$, the optical density was measured at $450 \mathrm{~nm}$.

\section{8. in vivo AZ505 administration and micro- computed tomography (CT) analysis}

Eight-week-old female mice (C57BL/6) were injected intraperitoneally with vehicle or AZ505 and the femurs were analyzed with SkyScan 1172 (SkyScan, Kontich, Belgium; 70 Kv, $141 \mu \mathrm{A}, 6.92$ pixel size). Trabecular bone was measured in the 1-mm-thick region starting $1 \mathrm{~mm}$ below the growth plate at thresholds of 89 minimum and 255 maximum. Bone parameters were calculated with the CTanalyzer program (version 1.7; SkyScan), and 3-dimensional images were obtained with the CT-volume software (version 1.11; SkyScan).

\section{Statistical analysis}

Statistical differences between the 2 groups were determined using Student's $t$-test. A $P$-value of less than 0.05 was considered significant.

\section{RESULTS}

\section{AZ505 promotes osteoblast differentiation in vitro}

We first assessed the effect of AZ505 on in vitro cultures of osteoblast precursors under an osteogenic condition. Preosteoblasts obtained from mice calvarial bones were cultured in a medium containing $\beta$-glycerophosphate and ascorbic acid to induce osteoblast differentiation in the presence or absence of AZ505. When cultures were stained for ALP activity, AZ505 resulted in an increase in the staining intensity in a dose-dependent manner at concentrations from $0.12 \mu \mathrm{M}\left(\mathrm{IC}_{50}\right)$ to $1.2 \mu \mathrm{M}$ (Fig. 1A). AZ505 did not exert cytotoxicity at concentrations up to $1.2 \mu \mathrm{M}$ in this culture condition (Fig. 1A). Quantitative ALP activity assays with cell lysates revealed a statistically significant increase in cells treated with AZ505 at $1.2 \mu \mathrm{M}$ (Fig. 1B). The matrix mineralization in the culture was evaluated by Alizarin red 

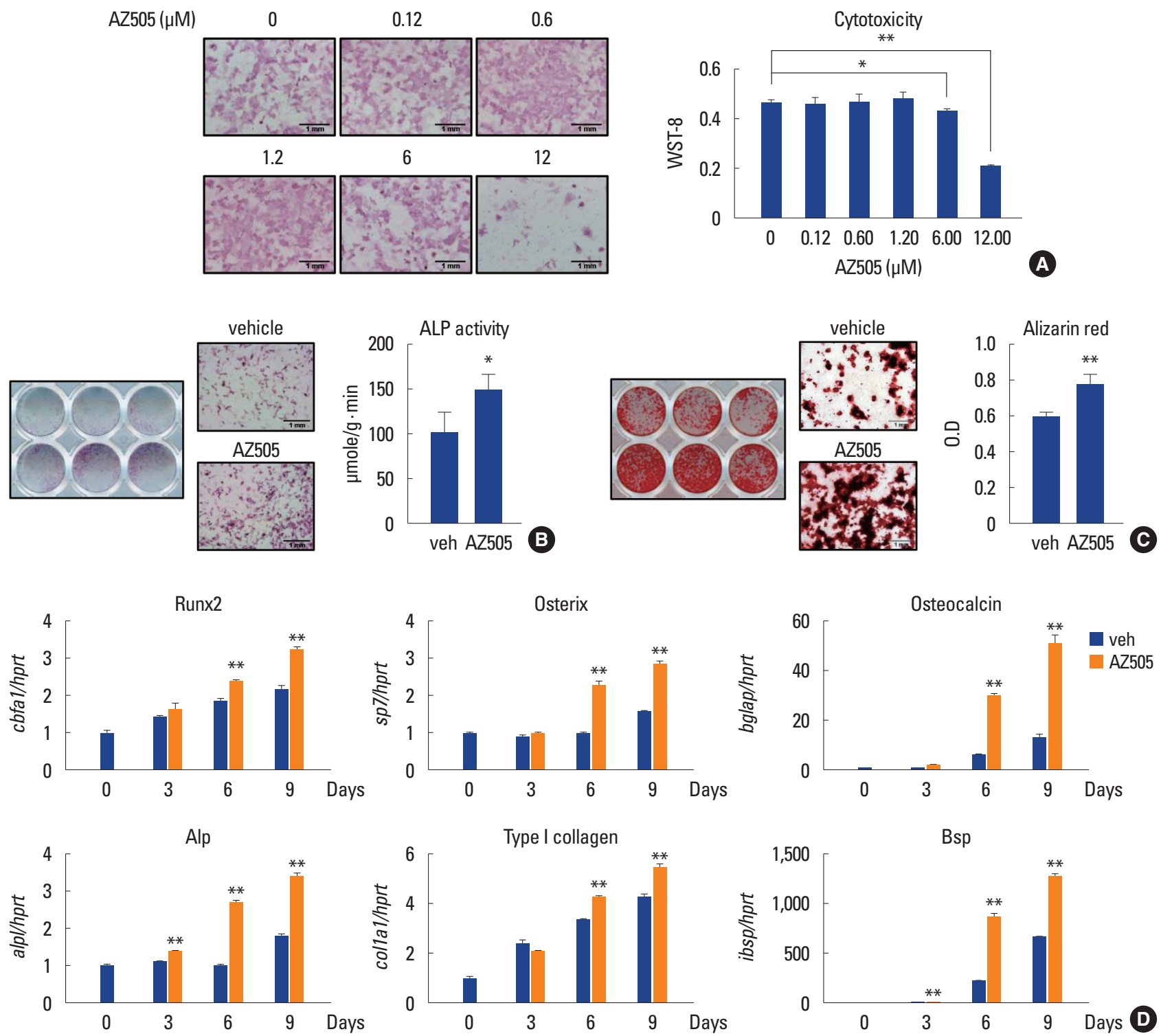

Fig. 1. AZ505 stimulates osteoblast differentiation in vitro. (A) Calvarial preosteoblasts were cultured in osteogenic medium with the indicated concentration of AZ505 for 3 days. Cells were subjected to alkaline phosphatase (ALP) staining ( $\times 40$ magnification) or cell viability assay. (B) Calvarial preosteoblasts were cultured in osteogenic medium for 3 days with $1.2 \mu \mathrm{M} \mathrm{AZ505}$ or vehicle, and ALP staining and ALP activity assay were performed. (C) Calvarial preosteoblasts were cultured in osteogenic medium for 9 days with $1.2 \mu \mathrm{M}$ AZ505 or vehicle and stained with Alizarin red ( $\times 40$ magnification). Alizarin red stain was quantified after solubilization with cetylpyridinium chloride. (D) Calvarial preosteoblasts were cultured in osteogenic medium containing either vehicle or $1.2 \mu \mathrm{M}$ AZ505 for the indicated days and subjected to reverse transcription-quantitative polymerase chain reaction. Data are presented as mean \pm standard deviation. ${ }^{* *} P<0.005$ vs. vehicle.

staining. Treatment with AZ505 (1.2 $\mu \mathrm{M})$ increased staining intensity (Fig. 1C).

With the results of ALP and Alizarin red staining, we next examined the effect of AZ505 on the expression of genes involved in osteoblast differentiation and matrix formation. The mRNA levels of transcription factors Runx2 (gene symbol cbfa1) and osterix (sp7), and matrix proteins colla- gen type I a1 (col1a1), osteocalcin (bglap), bone sialoprotein (ibsp), and ALP (alpl) were quantitatively measured by real-time PCR. AZ505 moderately increased mRNA levels of $c b f a 1$ and col1a1 while greatly upregulating mRNA levels of bglap, sp7, ibsp, and alpl (Fig. 1D). These results indicate that AZ505 promoted the osteoblast differentiation and mineral apposition in this calvarial preosteoblast cul- 

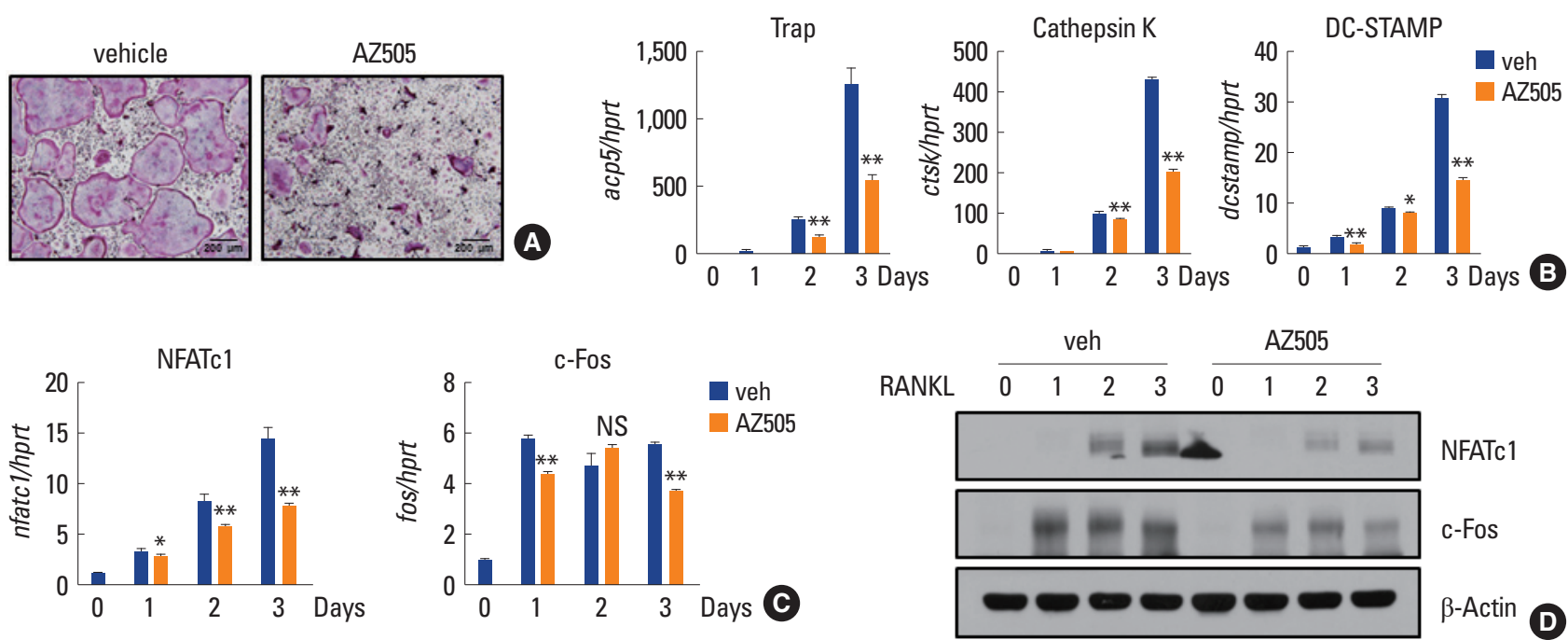

Fig. 2. AZ505 inhibits osteoclast differentiation in vitro. (A) Bone marrow-derived macrophages (BMMs) were cultured in the presence of macrophage colony-stimulating factor (M-CSF) and receptor activator of nuclear factor- $\kappa B$ ligand (RANKL) with or without $1.2 \mu \mathrm{M}$ AZ505 for 6 days and stained for tartrate-resistant acid phosphatase (TRAP) ( $\times 100$ magnification). (B, C) BMMs were cultured in the presence of M-CSF and RANKL with or without AZ505 for the indicated days and subjected to reverse transcription-quantitative polymerase chain reaction. (D) Protein lysates were prepared from cells cultured for the indicated days with M-CSF and RANKL in the presence or absence of AZ505. Western blot was performed using antibodies for nuclear factor of activated T cells (NFATc1), c-Fos and $\beta$-actin. Data are presented as mean \pm standard deviation. NS, not significant. ${ }^{*} P<0.05 .{ }^{*} P<0.005$ vs. vehicle.

ture and suggest that this SMYD2 inhibitor may have a bone anabolic function.

\section{AZ505 inhibits osteoclast differentiation in vitro}

We next investigated the effect of AZ505 treatment on osteoclast differentiation. Primary BMMs derived from mouse bone marrow cells were used as osteoclast precursor cells and cultured in the presence of M-CSF and RANKL with or without AZ505. When the culture was stained for TRAP activity, AZ505 was found to decrease the generation of multinucleated TRAP-positive osteoclasts (Fig. 2A). Consistent with the TRAP staining result, the upregulation of TRAP mRNA level by RANKL was attenuated in cells treated with AZ505 (Fig. 2B). The mRNA levels of other osteoclast marker genes, cathepsin $\mathrm{K}$ (ctsk) and dendritic cellspecific transmembrane protein (dcstamp), were also lower in AZ505-treated cells (Fig. 2B). The expression of these genes is controlled by the transcription factors NFATC1 and c-Fos, which have been shown to be essential for osteoclast differentiation. $[19,20]$ Therefore, we analyzed the mRNA and protein levels of NFATc1 and c-Fos in the osteoclastogenic cultures treated with AZ505. Both the mRNA and protein levels of NFATc1 were significantly lower in
AZ505-treated cultures (Fig. 2C, D). AZ505 also affected cFos levels to an extent much weaker than its effect on NFATc1 (Fig. 2C, D). These results demonstrate that AZ505 can suppress osteoclast differentiation by interfering with the NFATC1 induction by RANKL.

\section{AZ505 induces bone loss in mice}

Given that AZ505 promoted osteoblast differentiation and suppressed osteoclast formation in the in vitro cultures (Fig. 1, 2), we next sought to assess the in vivo effect of this chemical on bone metabolism by intraperitoneal injection into mice. Eight-week-old female mice were treated with 5 $\mathrm{mg} / \mathrm{kg}$ AZ505 daily for 4 weeks and micro-CT analysis was performed. To our surprise, femurs of the AZ505-treated group showed much lower trabecular bone mass than those of the vehicle-treated control group (Fig. 3A). Quantitative analyses revealed a great reduction in bone volume per tissue volume and trabecular number in AZ505treated mice (Fig. 3B). Trabecular separation was accordingly increased by AZ505 administration (Fig. 3B). These effects were observed even at $0.1 \mathrm{mg} / \mathrm{kg} /$ day dose administered only for 2 weeks (Fig. 3C), indicating a strong potency of this chemical on bone metabolism. 

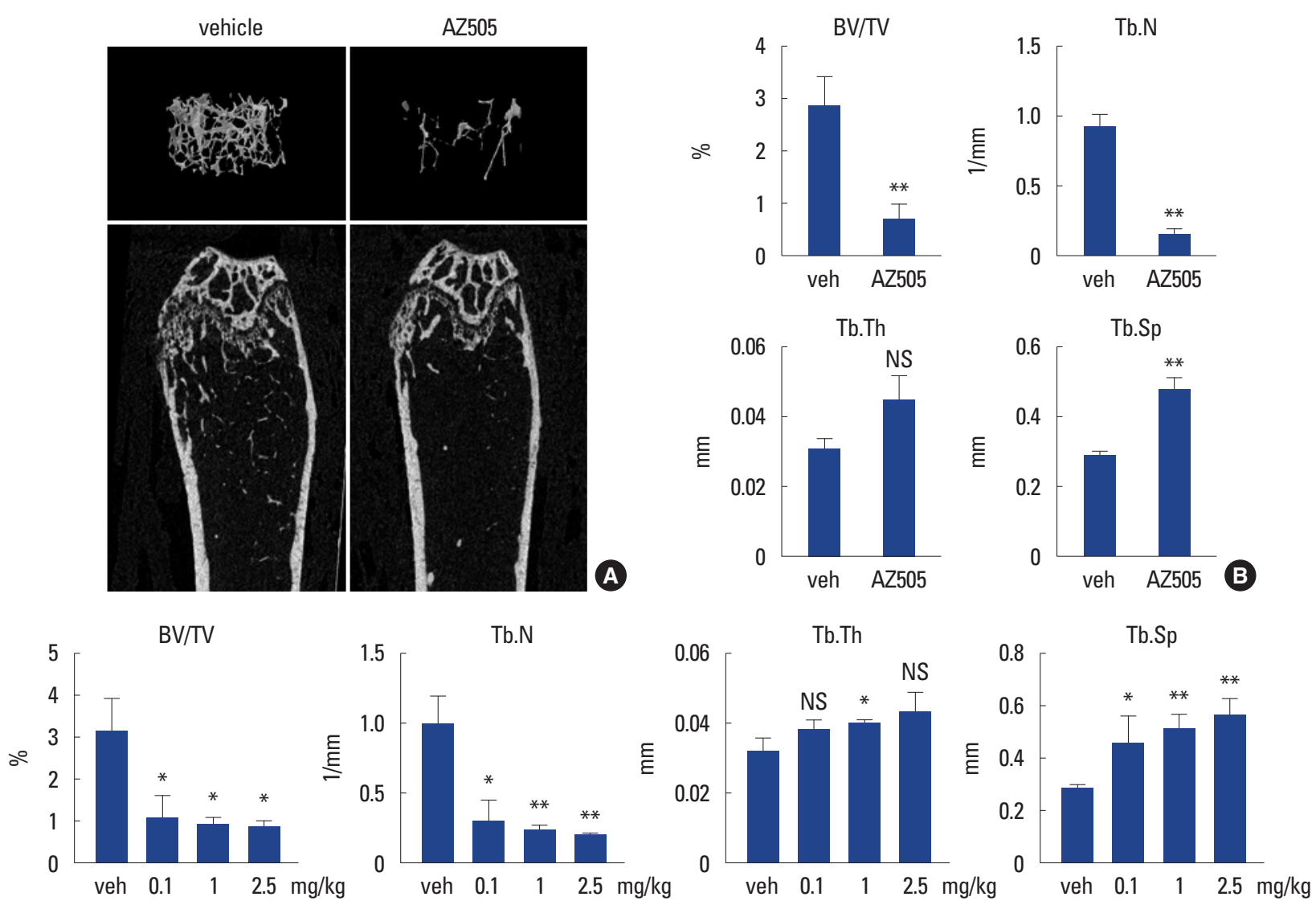

Fig. 3. AZ505 reduces trabecular bone mass in mice. (A, B) The 8-week-old female mice were injected intraperitoneally with vehicle or $5 \mathrm{mg} / \mathrm{kg}$ AZ505 for 4 weeks daily, and the femurs were subjected to micro-computed tomography (CT) analysis. (A) Representative 3-dimensional images of trabecular bone and coronal 2-dimensional images are shown. Bone parameters of trabecular bone volume per tissue volume (BV/TV), trabecular number (Tb.N), trabecular thickness (Tb.Th), and trabecular separation (Tb.Sp) were analyzed with a micro-CT analysis program (vehicle, $\mathrm{N}=4$; AZ505, N =5). (C) The 8-week-old female mice were intraperitoneally injected with vehicle or AZ505 (0.1, 1, $2.5 \mathrm{mg} / \mathrm{kg})$ for 2 weeks daily. The femurs were analyzed by micro-CT $(\mathrm{N}=3)$. Data are presented as mean \pm standard deviation. ${ }^{*} P<0.05 .{ }^{* *} P<0.005$ vs. vehicle.

\section{AZ505 potently stimulates RANKL expression}

As the in vivo result was in contradiction to the expectation derived from in vitro data, we next examined the possibility that AZ505 regulates the expressions of RANKL and osteoprotegerin (OPG) in osteoblasts. The mRNA levels of RANKL (tnfff11) and OPG (tnfsf11b) of calvarial preosteoblasts during osteogenic differentiation were assessed by quantitative real-time PCR. AZ505 increased tnfsf11 levels about 4-fold (Fig. 4A). The mRNA level of tnfsf11b was decreased by AZ505 treatment (Fig. 4B). These changes led to about 5 -fold increase in the ratio of tnfsf11/tnfsf11b by AZ505 (Fig. 4C). The strong increase in the RANKL/OPG ratio by AZ505 may explain the great reduction in bone mass of the mice treated with AZ505. Consistently, co-cultures of osteoblasts and bone marrow cells in the presence of 1,25-dihydroxy-vitamin D3 and prostaglandin $E_{2}$ dis- played an increase in osteoclasts number by AZ505-treatment (Fig. 4D).

\section{DISCUSSION}

An approach frequently utilized in finding drug candidates with therapeutic potential for osteoporosis is screening chemicals with in vitro cultures of osteoblasts or osteoclasts. By the cell culture-based screening, we found that AZ505 has a potent stimulatory effect on osteogenesis and inhibitory effect on osteoclastogenesis from precursors of osteoblasts and osteoclasts, respectively. With these desirable dual effects, we expected a strong positive outcome of increased bone mass in mice treated with AZ505. To our surprise, AZ505 severely reduced bone mass of femurs, which could be attributed, at least in part, to the stimulation of 

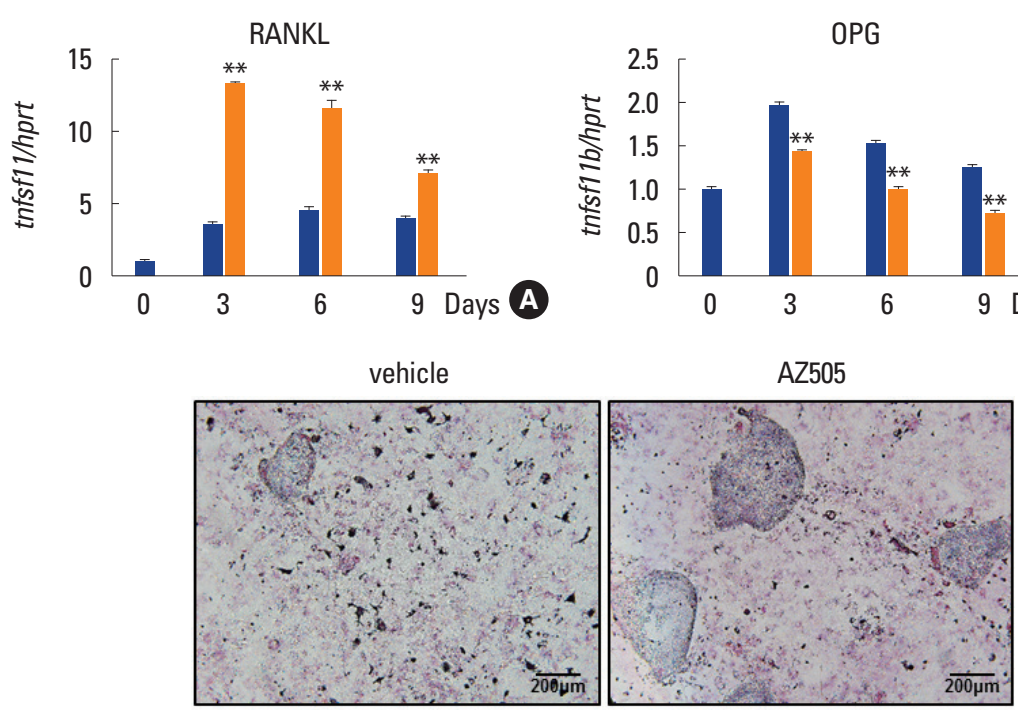
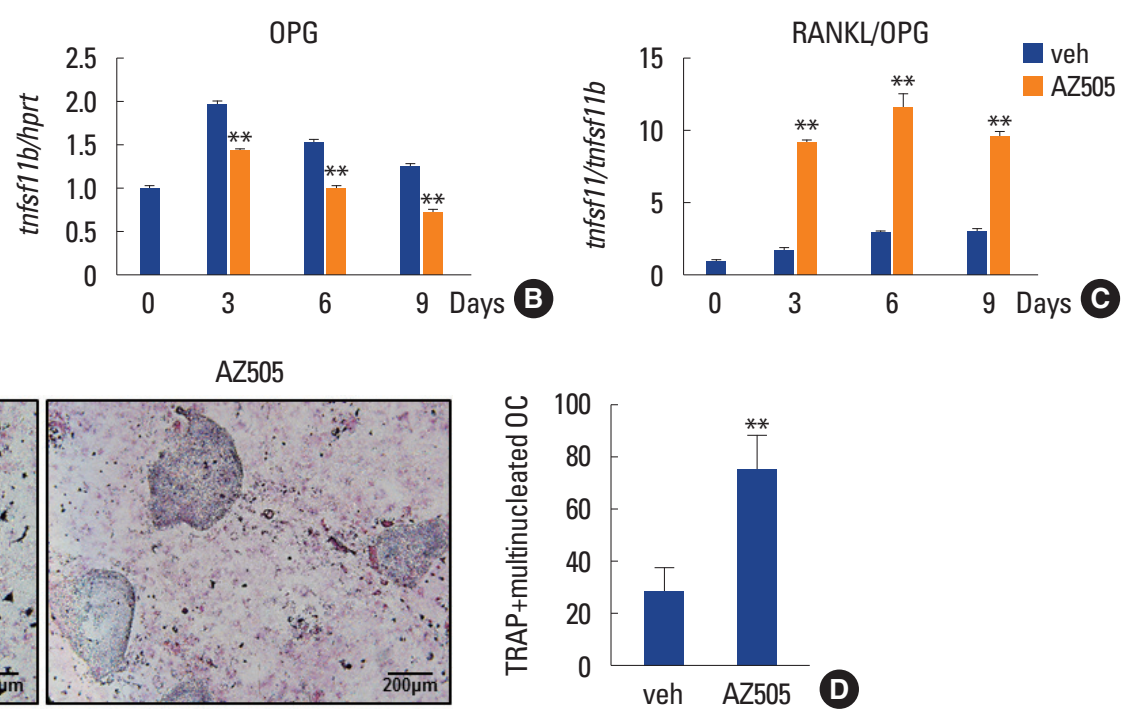

Fig. 4. Receptor activator of nuclear factor-kB ligand (RANKL) expression is induced by AZ505. (A-C) Calvarial preosteoblasts were cultured in osteogenic medium containing either vehicle or AZ505 $(1.2 \mu \mathrm{M})$ for the indicated days. The mRNA levels of tnfff11 and tnfsf11 b were analyzed by reverse transcription-quantitative polymerase chain reaction and the RANKL to osteoprotegerin ratio was calculated. (D) Calvarial preosteoblasts and bone marrow cells were co-cultured for 10 days with $1.2 \mu \mathrm{M}$ AZ505 or vehicle and stained for tartrate-resistant acid phosphatase (TRAP) ( $\times 100$ magnification). TRAP ${ }^{+}$multinucleated osteoclasts were counted. Data are presented as mean \pm standard deviation. ${ }^{* *} P<0.005$ vs. vehicle.

RANKL production. Our result showing detrimental effect in vivo despite promising positive effect in vitro signifies the importance of in vivo verification in development of osteoporosis therapeutics post in vitro cell-based screening.

In our study, AZ505 enhanced osteoblast differentiation and mineralization. Bone morphogenetic proteins (BMPs) are one of the representative osteogenesis inducers. There have been reports that show a potential link between BMP signaling and protein methyl transferases. In the study by Gao et al. [21], BMP type II receptor was shown to be methylated by SMYD2 in its kinase domain and the methylation led to enhanced BMP signaling in HaCaT keratinocytes. In addition, AZ505 suppressed the phosphorylation of Smad1/5 induced by BMP2.[21] The inhibitory effect of AZ505 on BMP2 signaling in $\mathrm{HaCaT}$ keratinocyte cells is contradictory to the stimulatory effect of AZ505 on osteogenesis observed in our study. To explain these contradictory observations, it is necessary to investigate where SMYD2 has a role in osteoblast differentiation and whether AZ505 affects BMP signaling in osteoblasts. AZ505 may affect targets in osteoblasts different from those in keratinocytes. Another possibility that cannot be ruled out might be the effects of AZ505 independent on its SMYD2 inhibitory function.

The bone loss induced by AZ505 in mice femurs might be attributed to the stimulation of RANKL expression in os- teoblasts (Fig. 4). Given that AZ505 inhibits SMYD2 methyltransferase, AZ505 might have affected RANKL gene expression through SMYD2-dependent protein methylation. Indeed, SMYD2 mediated H3K36 dimethylation in $\mathrm{J774}$ macrophages to regulate TNF- $\alpha$ expression.[4] In addition, SMYD2 methylated STAT3 and p65 transcription factors, promoting the proliferation of triple-negative breast cancer cell lines.[13] In analogy, it can be postulated that SMYD2 methylate transcription factors or histones were recruited to RANKL gene promoter. A potential candidate transcription factor is Runx2 as Runx2 was shown to increase RANKL expression in bone-metastatic breast and prostate cancer cells.[22] Whether Runx2 protein is methylated and whether Runx2 methylation regulates its activity have not been revealed yet.[23] However, Runx1 has been shown to be methylated at lysine by mixed-lineage leukemia and SUV39H1.[24-27] Similarly, Runx2 may be regulated by SMYD2-mediated methylation. An alternative possibility is that histone methylation at Runx2 promoter is mediated by SMYD2 and this methylation suppresses Runx2 expression. While evidence for this notion is lacking, demethylation of $\mathrm{H} 3 \mathrm{~K} 27$ by Jumonji domain-containing 3 was shown to increase Runx2 expression.[28]

Osteoclast activity is required for proper bone development and tooth eruption.[29] The deficit in formation and/ 
or function of osteoclasts cause osteopetrosis.[30,31] To ameliorate osteopetrosis, bone marrow transplantation is performed in expectation of transplanted hematopoietic cells supplying osteoclast precursors.[32-34] Cell therapy like bone marrow transplantation however has a disadvantage due to its risk of side effects including graft versus host disease. Achieving osteoclast stimulatory effects with small-molecule chemicals or lipids may be a more convenient and inexpensive strategy in treatment of osteopetrosis. Our results showing a great induction of RANKL and subsequent bone loss in AZ505-treated mice suggest a potential use of this chemical on osteopetrotic diseases.

\section{DECLARATIONS}

\section{Funding}

This study was supported by research funding from Korean Society for Bone and Mineral Research and a grant from the National Research Foundation of Korea (NRF2020R1A2C2010082).

\section{Ethics approval and consent to participate}

All animal studies were approved by the Animal Care and Use Committee of Seoul National University.

\section{Conflict of interest}

No potential conflict of interest relevant to this article was reported.

\section{ORCID}

Min-Kyoung Song https://orcid.org/0000-0002-2887-3909

Suhan Jung https://orcid.org/0000-0002-4301-9065

Jun-Oh Kwon https://orcid.org/0000-0001-5454-3391

Min Kyung Kim https://orcid.org/0000-0002-6512-0615

Hong-Hee Kim https://orcid.org/0000-0002-1411-6106

\section{REFERENCES}

1. Jeffries MA. The development of epigenetics in the study of disease pathogenesis. Adv Exp Med Biol 2020;1253:5794. https://doi.org/10.1007/978-981-15-3449-2_2.

2. Cho YD, Ryoo HM. Trans-differentiation via epigenetics: $A$ new paradigm in the bone regeneration. J Bone Metab 2018;25:9-13. https://doi.org/10.11005/jbm.2018.25.1.9.

3. Yi X, Jiang XJ, Fang ZM. Histone methyltransferase SMYD2: ubiquitous regulator of disease. Clin Epigenetics 2019;11: 112. https://doi.org/10.1186/s13148-019-0711-4.

4. Parmar N, Chandrakar P, Kar S. Leishmania donovani subverts host immune response by epigenetic reprogramming of macrophage M(Lipopolysaccharides + IFN- $\gamma$ )/M(IL-10) polarization. J Immunol 2020;204:2762-78. https://doi. org/10.4049/jimmunol.1900251.

5. Hamamoto R, Saloura V, Nakamura Y. Critical roles of nonhistone protein lysine methylation in human tumorigenesis. Nat Rev Cancer 2015;15:110-24. https://doi.org/10.1038/ nrc3884.

6. Huang J, Perez-Burgos L, Placek BJ, et al. Repression of p53 activity by Smyd2-mediated methylation. Nature 2006; 444:629-32. https://doi.org/10.1038/nature05287.

7. Scoumanne A, Chen X. Protein methylation: a new mechanism of p53 tumor suppressor regulation. Histol Histopathol 2008;23:1143-9. https://doi.org/10.14670/hh-23. 1143.

8. Cho HS, Hayami S, Toyokawa G, et al. RB1 methylation by SMYD2 enhances cell cycle progression through an increase of RB1 phosphorylation. Neoplasia 2012;14:476-86. https:// doi.org/10.1593/neo.12656.

9. Zhang X, Tanaka K, Yan J, et al. Regulation of estrogen receptor a by histone methyltransferase SMYD2-mediated protein methylation. Proc Natl Acad Sci U S A 2013;110: 17284-9. https://doi.org/10.1073/pnas.1307959110.

10. Jiang Y, Trescott L, Holcomb J, et al. Structural insights into estrogen receptor a methylation by histone methyltransferase SMYD2, a cellular event implicated in estrogen signaling regulation. J Mol Biol 2014;426:3413-25. https://doi. org/10.1016/j.jmb.2014.02.019.

11. Ferguson AD, Larsen NA, Howard T, et al. Structural basis of substrate methylation and inhibition of SMYD2. Structure 2011;19:1262-73. https://doi.org/10.1016/j.str.2011.06.011.

12. Fabini E, Manoni E, Ferroni $C$, et al. Small-molecule inhibitors of lysine methyltransferases SMYD2 and SMYD3: current trends. Future Med Chem 2019;11:901-21. https:// doi.org/10.4155/fmc-2018-0380.

13. Li LX, Zhou JX, Calvet JP, et al. Lysine methyltransferase SMYD2 promotes triple negative breast cancer progression. Cell Death Dis 2018;9:326. https://doi.org/10.1038/ s41419-018-0347-x.

14. Li LX, Fan LX, Zhou JX, et al. Lysine methyltransferase SMYD2 promotes cyst growth in autosomal dominant polycystic kidney disease. J Clin Invest 2017;127:2751-64. https:// 
doi.org/10.1172/jci90921.

15. Kim H, Kim B, II Kim S, et al. S100A4 released from highly bone-metastatic breast cancer cells plays a critical role in osteolysis. Bone Res 2019;7:30. https://doi.org/10.1038/ s41413-019-0068-5.

16. Park KH, Gu DR, Kim MS, et al. Inhibitory effect of rosae multiflorae fructus extracts on the receptor activator of NF-KB ligand-induced osteoclastogenesis through modulation of P38- and $\mathrm{Ca}(2+)$-mediated nuclear factor of activated T-cells cytoplasmic 1 expression. J Bone Metab 2020; 27:53-63. https://doi.org/10.11005/jbm.2020.27.1.53.

17. Kim MK, Kwon JO, Song MK, et al. Salt-inducible kinase 1 regulates bone anabolism via the CRTC1-CREB-Id1 axis. Cell Death Dis 2019;10:826. https://doi.org/10.1038/s41419019-1915-4.

18. Song MK, Park C, Lee YD, et al. Ga12 regulates osteoclastogenesis by modulating NFATc1 expression. J Cell Mol Med 2018;22:849-60. https://doi.org/10.1111/jcmm.13370.

19. Takayanagi $H$, Kim S, Koga $T$, et al. Induction and activation of the transcription factor NFATc1 (NFAT2) integrate RANKL signaling in terminal differentiation of osteoclasts. Dev Cell 2002;3:889-901. https://doi.org/10.1016/s1534-5807(02) 00369-6.

20. Grigoriadis $A E$, Wang ZQ, Cecchini MG, et al. c-Fos: a key regulator of osteoclast-macrophage lineage determination and bone remodeling. Science 1994;266:443-8. https:// doi.org/10.1126/science.7939685.

21. Gao S, Wang Z, Wang W, et al. The lysine methyltransferase SMYD2 methylates the kinase domain of type II receptor BMPR2 and stimulates bone morphogenetic protein signaling. J Biol Chem 2017;292:12702-12. https://doi.org/ 10.1074/jbc.M117.776278.

22. Kim B, Kim H, Jung S, et al. A CTGF-RUNX2-RANKL axis in breast and prostate cancer cells promotes tumor progression in bone. J Bone Miner Res 2020;35:155-66. https://doi. org/10.1002/jbmr.3869.

23. Blumenthal E, Greenblatt S, Huang G, et al. Covalent modifications of RUNX proteins: structure affects function. Adv Exp Med Biol 2017;962:33-44. https://doi.org/10.1007/ 978-981-10-3233-2_3.

24. Huang $G$, Zhao X, Wang $L$, et al. The ability of MLL to bind RUNX1 and methylate $\mathrm{H} 3 \mathrm{~K} 4$ at PU.1 regulatory regions is impaired by MDS/AML-associated RUNX1/AML1 mutations. Blood 2011;118:6544-52. https://doi.org/10.1182/blood2010-11-317909.

25. Koh CP, Wang CQ, Ng CE, et al. RUNX1 meets MLL: epigenetic regulation of hematopoiesis by two leukemia genes. Leukemia 2013;27:1793-802. https://doi.org/10.1038/leu. 2013.200.

26. Herglotz J, Kuvardina ON, Kolodziej S, et al. Histone arginine methylation keeps RUNX1 target genes in an intermediate state. Oncogene 2013;32:2565-75. https://doi. org/10.1038/onc.2012.274.

27. Chakraborty S, Sinha KK, Senyuk V, et al. SUV39H1 interacts with AML1 and abrogates AML1 transactivity. AML1 is methylated in vivo. Oncogene 2003;22:5229-37. https:// doi.org/10.1038/sj.onc.1206600.

28. Yang D, Okamura H, Nakashima Y, et al. Histone demethylase Jmjd3 regulates osteoblast differentiation via transcription factors Runx2 and osterix. J Biol Chem 2013;288: 33530-41. https://doi.org/10.1074/jbc.M113.497040.

29. Kong $Y Y$, Yoshida $H$, Sarosi I, et al. OPGL is a key regulator of osteoclastogenesis, lymphocyte development and lymph-node organogenesis. Nature 1999;397:315-23. https://doi.org/10.1038/16852.

30. Tondravi MM, McKercher SR, Anderson K, et al. Osteopetrosis in mice lacking haematopoietic transcription factor PU.1. Nature 1997;386:81-4. https://doi.org/10.1038/ $386081 a 0$.

31. Lacey DL, Timms E, Tan HL, et al. Osteoprotegerin ligand is a cytokine that regulates osteoclast differentiation and activation. Cell 1998;93:165-76. https://doi.org/10.1016/ s0092-8674(00)81569-x.

32. Coccia PF, Krivit W, Cervenka J, et al. Successful bone-marrow transplantation for infantile malignant osteopetrosis. N Engl J Med 1980;302:701-8. https://doi.org/10.1056/ nejm198003273021301.

33. Sorell M, Kapoor N, Kirkpatrick D, et al. Marrow transplantation for juvenile osteopetrosis. Am J Med 1981;70:12807. https://doi.org/10.1016/0002-9343(81)90839-1.

34. Orchard PJ, Fasth AL, Le Rademacher J, et al. Hematopoietic stem cell transplantation for infantile osteopetrosis. Blood 2015;126:270-6. https://doi.org/10.1182/blood-201501-625541. 
